\title{
PSICOPROFILAXIS EN EL PARTO EN PRESENTACION DE PELVIS
}

\author{
Dr. Guillermo Navas-Angel*
}

El hecho fundamental en el parto en presentación de pelvis, es la delimitación entre la eutocia y la distocia de este tipo de parto. Numerosos autores conceptúan que el parto en presentación de pelvis debe ser por la vía alta, mientras que otros, no menos numerosos, están seguros de que el parto en presentación de pelvis es tan normal como el de la presentación de vértice.

Nosotros, colocándonos en un terreno ecléctico, con base en nuestra experiencia profesional, consideramos que este tipo de parto que nos ocupa está justamente en el límite entre la eutocia y la distocia. Participa, en efecto, el feto en presentación de pelvis, de la situación longitudinal que es normal y cuando el tipo de presentación es de pelvis franca o incompleta el tamaño del polo pélvico es semejante en tamaño, consistencia y forma al de la cabeza fetal.

Con las premisas anteriores, la solución del parto en presentación de pelvis requiere un estudio detenido y pormenorizado de todos los factores que intervienen en él, a saber: paridad, pelvimetría, tamaño del feto, edad del embarazo, estado del cueIlo, integridad de las membranas o antigüedad en su ruptura, características de las partes blandas, normali- dad de la contracción uterina, estado psíquico de la paciente, etc. Recientemente los autores Zatuchni y Andros del Departamento de Obstetricia del Temple University Medical Center de Philadelphia en artículo publicado en el American Journal of Obstetric and Ginecology Sept. 15/ 65 proponen una calificación de los casos en presentación de pelvis basados en los datos anteriormente enunciados, obtenidos en el momento del ingreso de la paciente a los servicios hospitalarios. Acogemos con entusiasmo la propuesta de los autores americanos por cuanto permite evaluar y establecer una conducta apropiada y definitiva en el momento oportuno. Este hecho tiene particular importancia en el terreno que nos ocupa de la psicoprofilaxis, para no insistir con métodos sugestivos y prolongar un trabajo de parto sin finalidad ninguna cuando todo haya de resolverse por cesárea.

Recientemente, en curso de postgraduados de la Universidad Nacional, tuvimos ocasión de hacer una revisión del tema de presentación de pelvis que nos permitió obtener, mediante la consulta de literatura nacional y extranjera, algunos datos de suma importancia como son la ma-

\footnotetext{
* Profesor de Obstetricia. Universidad Nacio-
} nal. 
yor frecuencia de este tipo de presentación en partos prematuros, la mayor incidencia de pelvis incompletas o francas en las primíparas y de pelvis completas en las multíparas. La mayor posibilidad de rupturas prematuras de membranas en los casos de pelvis completas, una tendencia a la dilatación fácil e incorporación del cuello en los casos de pelvis francas junto con la posibilidad de dilatación incompleta y falta de borramiento total en las completas, duración del trabajo de parto sensiblemente igual al de los casos de presentaciones de vértice con variación en la duración del período expulsivo entre los dos tipos principales de presentaciones de pelvis con aumento en tiempo para las incompletas, compensado con una mejor preparación de los tejidos blandos del canal pelvigenital debido a la mejor regularidad del polo que desciende.

El encajamiento tardio y la presión insuficiente que ejercen los polos pélvicos sobre el cuello uterino y los con frecuencia un mal desencadenamiento del círculo vicioso del parto por ausencia del reflejo de Fergusson. Este hecho provoca, a su vez( una prolongación del pre-parto y una demora en la dilatación inicial del cuello uterino que con frecuencia obliga a la regularización de la motilidad uterina con soluciones ocitócicas.

El concepto de que el parto es una lucha entre fuerzas y resistencias tiene, en el caso del parto en presentación de pelvis, una vigencia absoluta puesto que mientras menores sean ios obstáculos que se opongan a la expulsión de la cabeza última, unánimemente considerada como el principal problema del parto en pelvis, mayor efectividad tendrán las fuerzas contráctiles uterinas, abdominales y perineales.

Por último, la idea, generalmente atendida en los medios obstétricos mundiales, de que mientras menos se intervenga en la atención de los partos en presentación de pelvis, los resultados son mejores, nos lleva a la conclusión de que la preparación psicoprofiláctica para la atención del parto es de una inmensa utilidad y representa una de las mejores adquisiciones de la obstetricia.

Ensayemos a continuación el análisis de las premisas anteriormente cnunciadas para llegar a demostrar la realidad de nuestro acerto.

La instrucción deparada a las pacientes obstétricas durante el período de gravidez nos permite darles una idea global y franca del problema de su parto en presentación de pelvis $y$ clemandar de ella el máximum de colaboración.

La necesidad de preservar la integridad de la bolsa de las aguas hasta la dilatación avanzada del cuello exige que durante las conferencias preparatorias se insista en evitar esfuerzos expulsivos o cambios de presión intra-abdominal en los últimos períodos del embarazo, la disminución de los contactos sexuales, el tratamiento oportuno de los estados infecciosos de vagina $y$ pelvis $y$ por sobre todo el abstenerse de pujar hasta cuando el médico no lo ordene, es decir, al alcanzar la dilatación completa. Estamos convencidos de que una paciente debidamente preparada conserva intacta la bolsa de las aguas por más tiempo que las parturientas ansiosas - sin preparación; y que mientras más permanezca íntegra la bolsa el cuello llega a una dilatación más completa y el comienzo del período expulsivo se hace con mayor normalidad. 
Siendo la frecuencia en las primigestantes sensiblemente igual a la de las multigestantes y considerando la mayor firmeza de las partes blandas de aquellas es fácil comprender que la práctica de la relajación y la atención y condicionamiento a todas las sugerencias que durante el curso se hagan, redundará en una menor oposición para la dilatación progresiva del cuello, de la vagina y del periné. En los casos de parto en presentación de vértice es común observar el descondicionamiento de las pacientes en el período de transición entre la dilatación y la expulsión, hecho que no se presenta en el parto en presentación de pelvis por la menor consistencia del polo pélvico y su descenso lento. Se puede, pues, pedir a la paciente esfuerzos oportunos, intensos y bien dirigidos para lograr el descenso y desprendimiento de las distintas partes del cuerpo fetal. Para la cabeza última el esfuerzo expulsivo debe comenzar con el desprendimiento de los hombros que es simultáneo con el encajamiento de la cabeza para lograr éste sin dificultad, y la ligera presión que el obstetra ejerce inmediatamente por encima del pubis para lograr la flexión de la cabeza suplirá la falta de efectividad de la contracción de la musculatura abdominal cuando ya el útero está casi completamente vacío.

Aún en el caso de una relajación absoluta del periné creemos aconsejable la práctica rutinaria de la episiotomía previa que facilitará los mecanismos de desprendimiento del feto y las posibles maniobras que haya necesidad de realizar para la extracción de los miembros superiores y de la cabeza última.

En nuestra práctica profesional en pacientes privadas y en los casos atendidos en el Instituto de Seguros Sociales hemos comprobado que el parto absolutamente espontáneo, es decir el que no requiere intervención ninguna del obstetra, se logra más fácilmente en las pacientes preparadas con psicoprofilaxis y que las mayores complicaciones se presentan en aquellas que conociendo la modalidad de su parto no lo encaran con el debido control por falta de preparación. Las analgesias regionales como anestesia de los pudendos, peridurales, caudales, etc. aplicadas con una dilatación de cinco a siete centímeiros en cuellos completamente borrados, bolsa íntegra, determinan una relajación absoluta del canal pelvigenital que facilita la expulsión del producto de la concepción en forma semejante a la lograda por los métodos sugestivos.

En resumen, la preparación psicoprofiláctica del parto evita las distocias de las partes blandas, la posibilidad de rupturas prematuras de membranas, aumenta la efectividad de las fuerzas de contracción y por último, pero no menos importante, suprime el efecto de drogas espasmolíticas o sedantes, no siempre inocuas para el feto, sobre todo en los casos de partos prematuros que son los más frecuentes en la presentación de pelvis.

Recōmendamos enfáticamente la atención de los partos en presentación de pelvis por los métodos de analgesia por psicoprofilaxis $y$, en su defecto, por las anestesias regionales.

Se calla, por entendido, que la conclucta anteriormente enunciada será para los casos en que la evaluación clínica de los factores del parto permita su terminación por vía baja y que el obstetra es tan esencial o más que la paciente en la solución de esıe problema obstétrico. 